\title{
EXERCISE INTENSITY AND THE PROTECTION FROM POSTPRANDIAL VASCULAR DYSFUNCTION IN ADOLESCENTS
}

B. Bond ${ }^{1}$, P. E. Gates ${ }^{2}$, S. R. Jackman ${ }^{3}$, L. M. Corless ${ }^{3}$, C. A. Williams ${ }^{1}$, and A. R. Barker ${ }^{1}$

${ }^{1}$ Children's Health and Exercise Research Centre, Sport and Health Science, College of Life and Environmental Sciences, University of Exeter, Exeter, EX1 2LU.

${ }^{2}$ Diabetes and Vascular Medicine, University of Exeter Medical School, Exeter, EX1 2LU.

${ }^{3}$ Sport and Health Science, College of Life and Environmental Sciences, University of Exeter, Exeter, EX1 2LU.

\section{Corresponding author:}

Dr Alan R. Barker

Children's Health and Exercise Research Centre

Sport and Health Sciences

College of Life and Environmental Sciences

University of Exeter

St Luke's Campus

Exeter

EX1 2LU

Tel: $44(0) 1392722766$

Fax: $44(0) 1392724726$

Email: A.R.Barker@exeter.ac.uk

Running title: Exercise intensity and adolescent vascular health

Key words: cardiovascular disease, endothelial function, postprandial lipaemia, young people 


\section{ABSTRACT}

Background: Acute exercise transiently improves endothelial function, and protects the vasculature from the deleterious effects of a high fat meal (HFM). We sought to identify whether this response is dependent on exercise intensity in adolescents. Methods: Twenty adolescents (10 male, $14.3 \pm$ $0.3 \mathrm{y})$ completed three 1 -day trials: 1) rest $(\mathrm{CON}) ; 2) 8 \times 1$ min cycling at $90 \%$ peak power with $75 \mathrm{~s}$ recovery (high-intensity interval exercise; HIIE); 3) cycling at $90 \%$ of the gas exchange threshold (moderate-intensity exercise; MIE) one hour before consuming a HFM (1.50 $\mathrm{g} \cdot \mathrm{kg}^{-1}$ fat). Macrovascular and microvascular endothelial function were assessed before and immediately after exercise, and three hours after the HFM by flow mediated dilation (FMD) and laser Doppler imaging (peak reactive hyperaemia; PRH). Results: FMD and PRH increased one hour after HIIE $(P<0.001, E S=1.20$ and $P=0.048, E S=0.56)$ but were unchanged after MIE. FMD and PRH were attenuated three hours after the HFM in CON $(P<0.001, E S=1.78$ and $P=0.02, E S=0.59)$. FMD remained greater three hours after the HFM in HIIE compared to MIE $(P<0.001, E S=1.47)$ and $\operatorname{CON}(P<0.001, E S=2.54)$, and in MIE compared to CON $(P<0.001, E S=1.40)$. Compared to CON, PRH was greater three hours after the HFM in $\operatorname{HIIE}(P=0.02, E S=0.71)$ and $\operatorname{MIE}(P=0.02$, $E S=0.84)$, with no differences between $\operatorname{HIIE}$ and $\operatorname{MIE}(P=0.72, E S=0.16)$. Plasma [triacylglycerol] and [total antioxidant status] were not different between trials. Conclusions: Exercise intensity plays an important role in protecting the vasculature from the deleterious effects of a HFM. Performing HIIE may provide superior vascular benefits than MIE in adolescent groups. 


\section{INTRODUCTION}

It is well established that the atherosclerotic process originates in childhood (58), and that cardiovascular disease (CVD) risk factors in youth are associated with the progression of atherosclerosis during adulthood (40). Endothelial dysfunction is a sentinel event in the progression of atherosclerosis, preceding the development of fatty streaks, and holds prognostic value in predicting CVD end points and patient mortality (59). Conduit artery endothelial function has been shown to be impaired in asymptomatic adolescents with CVD risk factors (16), whilst microvascular function is also impaired in children with clustered CVD risk (36). The ingestion of a high fat meal (HFM) causes a transient period of macro- and micro-vascular dysfunction $(5,54,70)$, and given the central role endothelial dysfunction plays in the atherosclerotic process (12), it is likely that repeat exposure of the vasculature to this environment has long-term implications for vascular health.

In adults, acute moderate and high-intensity exercise have transient benefits on macrovascular endothelial function in the fasted and postprandial state $(30,70)$, with the benefits more pronounced following high-intensity exercise possibly due to favourable changes in total antioxidant status (70). Prior exercise has also been shown to protect the microvasculature from the deleterious effects of a high fat meal in adults (26). In children, cross-sectional evidence suggests that high-intensity exercise may have a positive effect on fasting vascular function (32). Additionally, a single bout of moderate-intensity exercise (54) and sprint interval exercise (53) has been shown to preserve postprandial macrovascular function the following day in adolescent boys. However, the total exercise stimulus in these two studies was not equivalent, and the authors did not include a measure of microvascular function. Therefore, it is currently unknown whether exercise intensity modulates the postprandial macro- and micro-vascular dysfunction observed after a HFM in adolescents, which may have important public health implications as much of the day may be spent in the postprandial state. Furthermore, it has recently been shown that performing even small amounts $(\sim$ 
$4 \mathrm{~min}$ ) of high-intensity exercise is superior than moderate-intensity exercise at modifying cardiometabolic risk factors in youth (15). Considering that few adolescents meet the current recommended minimum of 60 min of moderate-intensity physical activity per day (50), and that habitual physical activity likely declines during adolescence $(37,69)$, it is pertinent to identify how small volumes of exercise can be optimised for vascular health in this group.

Given the above, this investigation sought to test the hypothesis that a single bout of high-intensity interval exercise (HIIE) provides superior protection of macrovascular function following a HFM compared to a work-matched bout of moderate-intensity exercise (MIE) in adolescents. We also assessed whether postprandial differences in macrovascular function were present at the microvascular level, and if differences in vascular function between trials were related to plasma [triacylglycerol] or total antioxidant status.

\section{METHODS}

Twenty 12 to 15 -year-old adolescents (10 males) volunteered to take part in this study. Participant assent and parental consent were obtained before participation in the project, which was approved by the institutional ethics committee. Exclusion criteria included the use of any medication or substance known to influence fat metabolism or vascular function.

Body mass, seated height and stature were measured to the nearest $0.1 \mathrm{~kg}$ and $0.1 \mathrm{~cm}$ respectively. Percentage body fat was estimated using triceps and subscapular skinfold thickness according to Slaughter et al. (57) and pubertal status was determined by a self-assessment of secondary sexual characteristics using adapted drawing of the five Tanner stages of pubic hair development (43).

\section{Visit 1: Fitness assessment}


The first visit included a validated combined ramp and supramaximal test to exhaustion to establish

111

112

113

maximal oxygen uptake $\left(\dot{V} \mathrm{O}_{2} \max \right)$ (6). Pulmonary $\dot{V} \mathrm{O}_{2}$ was monitored throughout (Cortex Metalyzer III B, Leipzig, Germany) and the gas exchange threshold was identified as the disproportionate increase in carbon dioxide production $\left(\dot{V} \mathrm{CO}_{2}\right)$ relative to $\dot{V} \mathrm{O}_{2}$. All exercise was performed on an electronically braked cycle ergometer (Lode Excalibur Sport, Groningen, the Netherlands).

\section{Visits 2-4: Exercise and postprandial measures}

Participants completed three experimental conditions, separated by approximately one week (Figure 1). Following $\mathrm{a} \sim 12 \mathrm{~h}$ overnight fast, participants were transported to the laboratory at 07:45 and rested for $15 \mathrm{~min}$ before providing a fasting fingertip capillary blood sample for plasma [triacylglycerol]. Participants then consumed $30 \mathrm{~g}$ of commercially available Corn Flakes with 130 $\mathrm{mL}$ of skimmed milk, which is unlikely to have influenced endothelial function (71).

At $08: 45$, participants rested in a darkened, temperature-controlled $\left(24^{\circ} \mathrm{C}\right)$ room for $10 \mathrm{~min}$ before the simultaneous assessment of macrovascular (flow mediated dilation (FMD)) and microvascular (laser Doppler perfusion imaging (LDI)) function. Immediately afterwards, capillary blood samples were obtained for plasma [triacylglycerol], [3-hydroxybutyrate] and total antioxidant status. These measurements were repeated one hour after exercise (but before the HFM) and three hours after the HFM in order to coincide with peak plasma [triacylglycerol] (67).

At 09:45, one hour after breakfast, participants either: 1) remained seated in the laboratory (CON); 2) performed $\sim 30$ min of continuous MIE at $90 \%$ of the gas exchange threshold; or 3) completed 23 min of HIIE. These trials were completed on separate days and in a randomised order. The HIIE bout consisted of a $3 \mathrm{~min}$ warm up at $20 \mathrm{~W}$, followed by $8 \times 1 \mathrm{~min}$ intervals at $90 \%$ of the peak power determined from the ramp test to exhaustion, interspersed with $75 \mathrm{~s}$ of recovery at $20 \mathrm{~W}$, 
before a 2 min cool down at $20 \mathrm{~W}$. The duration of the MIE trial was calculated to match the total work performed during the HIIE bout for each participant. Participants provided a rating of perceived exertion (RPE) (73) in the final $10 \mathrm{~s}$ of exercise. Participants also completed the 16-point Physical Activity Enjoyment Scale (PACES) (44) immediately after exercise cessation. After their final exercise trial, each participant was asked to identify which exercise bout they preferred. Plasma [triacylglycerol] and total antioxidant status were assessed one hour after the exercise/rest condition. Plasma [3-hydroxybutyrate] was also assessed as a marker of hepatic fatty acid oxidation and very low-density lipoprotein (VLDL) secretion (27). Participants then consumed a milkshake of 3 parts Cornish ice cream and one part double cream between 10:45 and 11:00, which provided $1.50 \mathrm{~g} \cdot \mathrm{kg}^{-1}\left(80 \mathrm{~kJ} \cdot \mathrm{kg}^{-1}\right)$ of fat in accordance with other postprandial investigations in this group (54, 67, 68) and our earlier work (11). Plasma [triacylglycerol] was assessed at hourly intervals during the three hour postprandial period. Participants remained seated in the laboratory throughout the postprandial period.

\section{Measures of vascular function}

FMD was measured using high resolution ultrasonography (Sequoia 512, Acuson, Siemens Corp, Aspen, USA) with a $13 \mathrm{MHz}$ linear array transducer and in accordance with recent guidelines (19, 61) and our earlier work (25). All FMD analyses were performed by primary investigator who was blinded to the condition. Baseline and post occlusion brachial artery diameter was assessed during end diastole using validated ECG-gating software (Medical Imaging Applications LLC, Coralvile USA) (41, 61). Baseline arterial diameter was measured for $1.5 \mathrm{~min}$. Endothelium-dependent vasodilation was calculated as the percentage increase in arterial diameter after a $5 \mathrm{~min}$ ischaemic stimulus (45) induced by rapid forearm pneumatic cuff inflation (Hokanson, Bellevue, USA) (8) to $220 \mathrm{mmHg}$. The area under the curve for estimated shear rate was calculated from the last $30 \mathrm{~s}$ of occlusion until the time of peak dilation $\left(\mathrm{SR}_{\mathrm{AUC}}\right)(61)$. To address concerns about the ratio-scaled 
FMD statistic (4), FMD was also allometrically scaled according to published guidelines (3). The between-day coefficient of variation for FMD was $10.5 \%$.

During the FMD protocol, microvascular function was simultaneously assessed using a laser Doppler perfusion imager (Periscan PIM II, Perimed, Järfälla, Sweden) at a reproducible point on the distal third of the forearm (20). High resolution data were collected at $4.33 \mathrm{~Hz}$, and then interpolated to $1 \mathrm{~s}$ averages before being smoothed using a $5 \mathrm{~s}$ moving average. Resting flux was measured over 2 min before cuff inflation. Peak reactive hyperaemia (PRH) was defined as the highest point after occlusion, and the between-day coefficient of variation was $16.2 \%$ for this variable.

\section{Blood analyses}

For each blood sample, $\sim 600 \mu \mathrm{L}$ of capillary blood was collected and centrifuged immediately at $13,000 \mathrm{~g}$ for $15 \mathrm{~min}$ at $4^{\circ} \mathrm{C}$. Plasma was then removed and stored at $-80^{\circ} \mathrm{C}$ for no more than one month. Plasma [triacylglycerol], [3-hydroxybutyrate] and total antioxidant status were quantified in duplicate by enzymatic, colorimetric methods using an assay kit according to the manufacturer's guidelines (Cayman Chemical Company, MI, USA). The within-batch coefficients of variation for plasma [triacylglycerol], [3-hydroxybutyrate] and total antioxidant status were 2.9, 3.8 and 4.2\% respectively. The total (TAUC) and incremental (IAUC) area under the curve analyses were performed using the time point immediately before the HFM for plasma [triacylglycerol], and the time point immediately before exercise for plasma [3-hydroxybutyrate] and total antioxidant status.

\section{Control of diet and exercise}

With parental supervision, participants were asked to replicate their evening meal prior to each laboratory visit. Participants also completed a food diary during the 48 hour period immediately preceding each visit, which were subsequently assessed for total energy and macronutrient intake 
(CompEat Pro, Nutrition Systems, UK). Participants were instructed to avoid strenuous exercise and wear a tri-axial accelerometer on their wrist (GENEActiv, Activinsights Ltd, Cambridge, UK) during the 48 hour prior to each visit. Time spent performing moderate to vigorous activity was determined using established cut points for paediatric groups (48).

\section{Statistical analyses}

Descriptive statistics were calculated using SPSS (version 19.0, Chicago, USA) and presented as mean \pm SD. Mean differences in descriptive statistics between boys and girls were analysed using independent samples $t$ tests. The mean differences in the physiological and perceptual responses of the boys and girls during HIIE and MIE were analysed using paired samples $t$ tests. Analysis of plasma [triacylglycerol], [3-hydroxybutyrate] and total antioxidant status, and parameters of macroand micro-vascular function were performed using a mixed model ANOVA with trial (CON, MIE, HIIE) and sex (male, female) as the main effects. For clarity, the main effects for time and condition are not discussed if the ANOVA output revealed a significant interaction effect. The inclusion of sex into the ANOVA model did not reveal a significant interaction effect for plasma [3hydroxybutyrate] and total antioxidant status or parameters of macro- and micro-vascular function. Data were subsequently pooled for these outcomes. Pairwise comparisons between means were interpreted using the $P$ value and standardised effect sizes $(E S)$ to document the magnitude of the effect using the thresholds: small (0.2), moderate (0.5) and large (0.8) (18). Relationships between changes in vascular outcomes and mechanistically important variables were explored using Pearson's correlations.

\section{RESULTS}

Baseline participant characteristics are presented in Table 1. The maturation status for boys and girls was as follows; Tanner stage $3, n=4$ and $n=1$; stage, $4 n=4$ and $n=8$; stage $5, n=2$ and $n=1$. No differences in energy intake, individual macronutrient contributions, or time spent performing 
227

228

moderate to vigorous physical activity were apparent for boys or girls during the 48 hour preceding each laboratory visit $(P>0.14, E S<0.20$; Table 2$)$.

Table 3 presents the physiological and perceptual data from the exercise trials. The highest $\dot{V} \mathrm{O}_{2}$ achieved during the HIIE condition equated to $93 \pm 5 \%$ and $96 \pm 5 \% \dot{V} \mathrm{O}_{2}$ max for boys and girls respectively. Average length of the MIE trial was $24.9 \pm 2.3 \mathrm{~min}$. Nine boys and nine girls indicated that they preferred the HIIE exercise bout.

\section{Blood analyses}

Mean differences in plasma [triacylglycerol] during the postprandial period are illustrated in Figure 2A. Mean fasted plasma [triacylglycerol] was lower across all trials in girls $(P=0.03, E S=0.96)$. There was no trial by sex interaction $(P=0.44)$ for TAUC-triacylglycerol, but there was a trend for TAUC-triacylglycerol to be lower in girls across all trials $(P=0.05)$. There was no trial by sex interaction $(P=0.58)$ for IAUC-triacylglycerol.

Mean differences in plasma [3-hydroxybutyrate] are illustrated in Figure 2B. A time by trial interaction $(P=0.04)$ was apparent for plasma [3-hydroxybutyrate], which was elevated three hours after the HFM in HIIE compared to $\mathrm{CON}(P=0.01, E S=0.59)$, with no differences between MIE and $\operatorname{CON}(P=0.16, E S=0.26)$ or HIIE and $\operatorname{MIE}(P=0.13, E S=0.29)$. An increase in TAUC plasma [3hydroxybutyrate] in HIIE was associated with lower TAUC-triacylglycerol $(P=0.01, r=0.61)$ but not for $\operatorname{MIE}(P=0.22, r=0.30)$.

Mean differences in total antioxidant status are provided in Figure 2C. There was no time by trial interaction $(P=0.53)$ or effect of trial $(P=0.88)$, but there was a main effect of time for total antioxidant status $(P=0.04)$. Mean total antioxidant status across conditions was lower after the 
HFM compared to baseline $(P=0.02, E S=0.39)$. Changes in total antioxidant status were not related to parameters of vascular function $(P>0.05$ and $r<0.2)$.

\section{Macrovascular function}

Differences in FMD between trials are presented in Figure 3A. There was a time by trial interaction $(P<0.001)$ for FMD. FMD was greater one hour after HIIE $(P<0.001, E S=1.20)$, but unchanged after $\operatorname{MIE}(P=0.22, E S=0.09)$ and $\operatorname{CON}(P=0.99, E S<0.01)$ compared to before exercise. Consequently, FMD was greater after HIIE compared to MIE $(P=0.002, E S=1.14)$ and CON $(P=0.002, E S=1.15)$, with no difference between MIE and $\operatorname{CON}(P=0.59, E S=0.15)$ one hour after exercise.

FMD was greater three hours after the HFM in HIIE compared to MIE $(P<0.001, E S=1.47)$ and $\operatorname{CON}(P<0.001, E S=2.54)$, and in MIE compared to $\operatorname{CON}(P<0.001, E S=1.40)$. FMD was attenuated after the HFM in $\mathrm{CON}(P<0.001, E S=1.78)$ compared to before the meal. FMD remained elevated after the HFM compared to baseline in HIIE $(P<0.001, E S=1.56)$. Differences in $\mathrm{SR}_{\mathrm{AUC}}$ between trials are provided in Figure 3B. Changes in FMD were not related to $\mathrm{SR}_{\mathrm{AUC}}$ in any trial. Consequently, FMD was not normalised for $\mathrm{SR}_{\mathrm{AUC}}$. There was no time by trial interaction for $\operatorname{SR}_{\mathrm{AUC}}(P=0.25)$, resting arterial diameter $(P=0.11$, Figure $3 \mathrm{C})$, or time taken to reach peak dilation $(P=0.37)$.

\section{Microvascular function}

Differences in PRH between trials are presented in Figure 3D. There was a time by trial interaction $(P=0.002)$ for PRH. PRH was greater one hour after HIIE $(P=0.004, E S=0.82)$ but unchanged after $\operatorname{MIE}(P=0.22, E S=0.26)$ and $\operatorname{CON}(P=0.27, E S=0.26)$. Compared to $\mathrm{CON}$, PRH was greater three hours after the HFM in HIIE $(P=0.02, E S=0.71)$ and MIE $(P=0.02, E S=0.84)$, with no difference between HIIE and MIE $(P=0.72, E S=0.16)$. PRH was attenuated three hours after the HFM in CON 
$(P=0.02, E S=0.59)$. There was no effect of trial $(P=0.15)$, time $(P=0.40)$, or a trial by time interaction $(P=0.27)$ for time taken to achieve PRH.

\section{DISCUSSION}

The novel findings from this study are: 1) macro- and micro-vascular function were enhanced one hour after HIIE compared to CON and MIE, and remained elevated three hours after a HFM; 2) a single bout of MIE did not alter macro- or micro-vascular function one hour after exercise, but prevented the decline in function observed three hours after a HFM; and 3) the interactions between exercise intensity and vascular function were independent of changes in plasma [triacylglycerol] or total antioxidant status. These data show for the first time that the effect of exercise on postprandial vascular function is dependent on exercise intensity. Specifically, macrovascular function after a HFM is preserved by MIE, and augmented by HIIE. These findings may have a clinically important public health message as a significant proportion of time is spent in the postprandial state, and endothelial function predicts cardiovascular events independently of conventional CVD risk factors (12).

The HFM reduced FMD by $21 \%$ in CON, which is consistent with other adolescent (54) and adult $(5,70,71)$ data. For the first time in adolescents, we provide evidence that a single bout of MIE performed one hour before a HFM may preserve endothelial function, and that an equivalent bout of HIIE not only prevents this attenuation but improves endothelial function despite no reduction in plasma [triacylglycerol]. Whilst the benefits of prior moderate-intensity (54) and sprint interval (53) exercise on postprandial macrovascular function have been shown to be unrelated to changes in plasma [triacylglycerol] in adolescents, we are the first to identify an independent effect of exercise intensity. Our findings concur with those reported by Tyldum et al. (70), however these authors identified that this protective effect of exercise performed the day before a HFM was related to an exercise-induced increase in antioxidant capacity, which we did not observe in this study. It is 
known that postprandial lipaemia impairs vascular function via oxidative stress (5), which may reduce nitric oxide bioavailability (72). FMD is considered to be largely nitric oxide dependent (28), but we did not observe an effect of exercise on total antioxidant status, or a relationship between FMD and total antioxidant status. However, Johnson et al. (35) also reported no relationship between post exercise FMD and oxidative stress, and this may be related to the limitation of a single measurement of oxidative stress rather than rate of antioxidant depletion (22). Furthermore, the exercise bouts in this study were performed one hour, compared to 16-18 hours (70), before the ingestion of the HFM, and thus the process(es) underlying the response in pro/antioxidant state are likely to be mechanistically different. Indeed a recent investigation failed to observe any changes in postprandial antioxidant status after MIE and HIE when exercise was performed one hour after a HFM (14). Additionally, we cannot account for the influence of training status on the changes in pro/antioxidant status following the exercise bouts (10). However, based upon recommended $\dot{V} \mathrm{O}_{2 \text { max }}$ cut off values for cardiometabolic health (1), 5 of the boys and 2 of the girls included in this study could be identified as "at risk", and the $\dot{V} \mathrm{O}_{2}$ max values observed in the present study were typically lower than those reported in trained groups (2).

Previous studies with healthy adults report that FMD either increases $(35,70)$, decreases $(22,35)$ or remains unaltered $(23,52)$ after a single bout of exercise, however these data are difficult to interpret due to inconsistencies in the intensity, duration and modality of exercise, and the timing of the FMD measurement(s) (22). The present study is the first to incorporate a work-matched exercise protocol in order to isolate the influence of exercise intensity on vascular function in adolescents, and our data show that FMD is increased one hour after HIIE but remains unaltered after MIE. In contrast, an exercise intensity dependent decrease in FMD has been shown immediately after cycling in adults (9), and exergaming in children (42). It is likely that this disparity is due to the timing of our FMD measure (one hour vs. immediately after exercise) as the FMD response post exercise is biphasic in nature (23). Indeed, it is thought that the temporary blunting of FMD 
observed after high-intensity, but not $\operatorname{MIE}(9,35,42)$, is the stimulus for subsequent improvements in FMD (47), however no study has yet identified the time course of the FMD response following work-matched exercise in adolescents.

Changes in FMD after exercise have been attributed to differences in baseline arterial diameter and shear rate (22). However, these remained unaltered between trials in the present study and there was no relationship between the magnitude of the FMD response and $\mathrm{SR}_{\mathrm{AUC}}$, which is consistent with existing data in children (62) and following exercise in adults (38). However, we did not quantify shear stress during the exercise bouts. Given that the exercise conditions were work-matched, it is likely that the disparate responses in FMD observed post exercise are related to the positive association between brachial artery shear and the intensity of cycling exercise $(29,63)$. This has been shown to play a leading role in modulating the post exercise FMD response $(64,65)$, probably due to an upregulation in endothelial nitric oxide synthase and subsequent increase in the bioavailability of nitric oxide (34). We are unable to partition out the influence of the HFM on the postprandial FMD response following MIE and HIIE. For example, it is possible that postprandial FMD could have been higher still following HIIE. However, considering that FMD has been demonstrated to return to baseline 2 hours post high-intensity exercise (35), and the lack of change in total antioxidant status in the present study, it would appear that the inclusion of a HFM 1 hour after exercise did not modulate the post exercise nitric oxide bioavailability. Further study is needed to confirm this.

A novel feature of this investigation was the simultaneous assessment of microvascular function during the FMD protocol. Whilst the endothelium only plays a part of the PRH response (20), impaired microvascular reactive hyperaemia is associated with elevated blood pressure (56), obesity (24), insulin resistance (33), and has been identified in healthy children with clustered CVD risk factors (36). Therefore, it follows that the assessment of PRH as a surrogate of microvascular 
342

343

344

345

346

347

348

349

350

351

352

353

354

355

356

357

358

359

360

361

362

363

364

365

366

367

function in the current study may provide useful information regarding vascular health in asymptomatic individuals. We observed a significant impairment in postprandial microvascular function in $\mathrm{CON}$, suggesting that a fatty meal presents a global challenge to the vasculature. This dysfunction was prevented in both exercise trials, but not in an intensity-dependent manner. To our knowledge, no other study has identified the effect of exercise intensity on subsequent postprandial microvascular function, however Gill et al. observed a similar protective effect of MIE performed the evening before a HFM in adults and this was endothelium-dependent (26).

Prior MIE (67) and HIIE (60) can attenuate postprandial lipaemia in adolescents, however we were unable to replicate these findings in this study, possibly due to our use of a one day protocol (74) and a short (three hour) postprandial observation period. It has been hypothesised that exerciseinduced changes in hepatic very low density lipoprotein (VLDL) output may explain some of the reduction in postprandial lipaemia after a HFM (39), particularly when the time between exercise cessation and consumption of the test meal is short due to the delay in the upregulation of lipoprotein lipase (55). Our data would appear to be consistent with this theory, as [3hydroxybutyrate] was elevated three hours after the HFM in HIIE compared to CON, and significantly correlated with the reduction in TAUC-triacylglycerol, suggesting a shift towards hepatic fatty acid oxidation rather than re-esterification and VLDL synthesis during the HIIE condition (27).

Repeated sprint cycling the day before a HFM has previously been demonstrated to preserve postprandial macrovascular function in adolescents (53). However, these authors reported that one third of the participants failed to complete the exercise protocol. In contrast, all participants in the present study completed the HIIE bout. Furthermore, our data indicate that HIIE was perceived to be more enjoyable than MIE for both boys and girls, despite a greater physiological stress. This is encouraging considering that adolescents rarely sustain exercise for longer than 10 minutes (50), 
therefore low-volume, high-intensity exercise may be a suitable method of optimising this pattern of activity provided that the exercise is not an "all-out" effort. Further work is needed to identify the long term adherence to a HIIE training intervention in this group, however preliminary evidence is promising (13). Indeed, our data add to a growing body of evidence which indicates that HIIE is a feasible and attractive alternative to MIE in adolescents $(11,21,49)$.

This is the first study to isolate the influence of exercise intensity on postprandial vascular function in adolescents. A further novelty of this study is the simultaneous assessment of microvascular function during the FMD protocol. However, our findings should be interpreted in light of a number of methodological considerations. Firstly, whilst post-occlusive reactive hyperaemia has been used as a marker of microvascular function in adolescents (51), the mechanisms underlying the PRH response to 5 minutes of ischaemia following exercise and a HFM are yet to be fully determined, but likely involve other pathways in addition to changes in endothelial function (20). However, postprandial microvascular function has been shown to be improved following exercise elsewhere and this was endothelium-dependent (26). Therefore, it is likely that some of the improvements observed in macrovascular endothelial function via FMD in the present study are present at the microvascular level. Secondly, we were unable to control for the menstrual cycle, which has been shown to influence FMD in women (31). The median stage of maturity (Tanner 4) suggests that some girls would be pre or post menarche (7), and whilst there was no significant interaction effect of sex on macro- or micro-vascular function in the present study, further work is necessary to explicitly establish whether sex influences this outcome in adolescents and in children. Thirdly, the HFM used in this study has limited ecological validity but provided a metabolic challenge in accordance with other postprandial investigations with adolescents $(11,54,66,68)$. This meal also provided an average of $35 \mathrm{~g}$ of sugar, which could plausibly have contributed to the postprandial responses (17), although this is equivocal (46). Future work is needed to identify how prior exercise can alter macro- and micro-vascular function following more habitual fat loads and feeding 
regimes. Finally, we were unable to determine endothelial-independent function via a sublingual spray of nitroglycerin (19), and this remains an area of future research.

\section{CONCLUSION}

Macro- and micro-vascular dysfunction occur in concert after a HFM in adolescents. We have shown that postprandial vascular function can be preserved after MIE, or improved after HIIE, and these changes were not related to plasma [triacylglycerol] or total antioxidant status. Whilst these findings cannot be extrapolated beyond healthy adolescents, they may have clinical importance as repeat impairment in endothelial function likely plays a key role in the development of CVD, which is known to have its origins in childhood (58). Future work is needed to assess the efficacy of different exercise intensities on postprandial endothelial function in adolescents with risk factors for CVD (e.g. obesity, type I diabetes). Finally, we also report here that HIIE was perceived to be more enjoyable than MIE, despite the greater physiological stress. Taken together, low-volume HIIE may be a feasible and attractive strategy to reduce CVD risk from an early age.

\section{Acknowledgements}

We thank the staff and participants at Blundell's School (Devon, UK) and Yasmin Pratt for their participation in this project.

\section{Funding}

This study was supported by the Physiological Society.

\section{Disclosures}

The authors have no competing interests to disclose 


\section{REFERENCES}

421

422 1. Adegboye AR, Anderssen SA, Froberg K, Sardinha LB, Heitmann BL, Steene-

423 Johannessen J, Kolle E, and Andersen LB. Recommended aerobic fitness level for metabolic 424 health in children and adolescents: a study of diagnostic accuracy. Br J Sports Med 45: 722-728, $425 \quad 2011$.

426 2. Armstrong N, and Barker AR. Endurance training and elite young athletes. Medicine and 427 sport science 56: 59-83, 2011.

428 3. Atkinson G, and Batterham AM. Allometric scaling of diameter change in the original 429 flow-mediated dilation protocol. Atherosclerosis 226: 425-427, 2013.

DH, Jones H, Tinken TM, and Green DJ. Is the ratio of flow-mediated dilation and shear rate a statistically sound approach to normalization in cross-sectional studies on endothelial function? $J$ Appl Physiol (1985) 107: 1893-1899, 2009.

KY, and Schwemmer M. Postprandial hypertriglyceridemia impairs endothelial function by enhanced oxidant stress. Atherosclerosis 155: 517-523, 2001. uptake in young people during a ramp cycle test to exhaustion. Br J Sports Med 45: 498-503, 2011. 7. Baxter-Jones ADG, Eisenmann JC, and Sherar LB. Controlling for maturation in 440 pediatric exercise science. Pediatric Exercise Science 17: 18-30, 2005.

441 8. Betik AC, Luckham VB, and Hughson RL. Flow-mediated dilation in human brachial 442 artery after different circulatory occlusion conditions. Am J Physiol Heart Circ Physiol 286: H442443 $448,2004$. 
DJ. Effects of exercise intensity on flow mediated dilation in healthy humans. Int J Sports Med 34:

446

447

448

449

450

451

452

453

454

455

456

457

458

459

460

461

462

463

464

465

466

467

468 409-414, 2013.

10. Bogdanis GC, Stavrinou P, Fatouros IG, Philippou A, Chatzinikolaou A, Draganidis D, Ermidis G, and Maridaki M. Short-term high-intensity interval exercise training attenuates oxidative stress responses and improves antioxidant status in healthy humans. Food and chemical toxicology : an international journal published for the British Industrial Biological Research Association 61: 171-177, 2013.

11. Bond B, Williams CA, Isic C, Jackman SR, Tolfrey K, Barrett LA, and Barker AR. Exercise intensity and postprandial health outcomes in adolescents. Eur J Appl Physiol 2014.

12. Bonetti PO, Lerman LO, and Lerman A. Endothelial dysfunction: a marker of atherosclerotic risk. Arterioscler Thromb Vasc Biol 23: 168-175, 2003.

13. Buchan DS, Ollis S, Young JD, Thomas NE, Cooper SM, Tong TK, Nie J, Malina RM, and Baker JS. The effects of time and intensity of exercise on novel and established markers of CVD in adolescent youth. Am J Hum Biol 23: 517-526, 2011.

14. Canale RE, Farney TM, McCarthy CG, and Bloomer RJ. Influence of acute exercise of varying intensity and duration on postprandial oxidative stress. Eur J Appl Physiol 114: 1913-1924, 2014.

15. Carson V, Rinaldi RL, Torrance B, Maximova K, Ball GD, Majumdar SR, Plotnikoff RC, Veugelers P, Boule NG, Wozny P, McCargar L, Downs S, Daymont C, Lewanczuk R, and McGavock J. Vigorous physical activity and longitudinal associations with cardiometabolic risk factors in youth. Int J Obes (Lond) 38: 16-21, 2014.

16. Celermajer DS, Sorensen KE, Gooch VM, Spiegelhalter DJ, Miller OI, Sullivan ID, Lloyd JK, and Deanfield JE. Non-invasive detection of endothelial dysfunction in children and adults at risk of atherosclerosis. Lancet 340: 1111-1115, 1992. 
Evidence for an independent and cumulative effect of postprandial hypertriglyceridemia and hyperglycemia on endothelial dysfunction and oxidative stress generation: effects of short- and long-term simvastatin treatment. Circulation 106: 1211-1218, 2002.

18. Cohen J. Statistical Power Analysis for the Behavioural Sciences. Hillsdale: Lawrence Erlbaum, 1988.

19. Corretti MC, Anderson TJ, Benjamin EJ, Celermajer D, Charbonneau F, Creager MA, Deanfield J, Drexler H, Gerhard-Herman M, Herrington D, Vallance P, Vita J, and Vogel R. Guidelines for the ultrasound assessment of endothelial-dependent flow-mediated vasodilation of the brachial artery: a report of the International Brachial Artery Reactivity Task Force. J Am Coll Cardiol 39: 257-265, 2002.

20. Cracowski JL, Minson CT, Salvat-Melis M, and Halliwill JR. Methodological issues in the assessment of skin microvascular endothelial function in humans. Trends in pharmacological sciences 27: 503-508, 2006.

21. Crisp NA, Fournier PA, Licari MK, Braham R, and Guelfi KJ. Adding sprints to continuous exercise at the intensity that maximises fat oxidation: implications for acute energy balance and enjoyment. Metabolism 61: 1280-1288, 2012.

22. Dawson EA, Green DJ, Cable NT, and Thijssen DH. Effects of acute exercise on flowmediated dilatation in healthy humans. J Appl Physiol (1985) 115: 1589-1598, 2013.

\section{Dawson EA, Whyte GP, Black MA, Jones H, Hopkins N, Oxborough D, Gaze D, Shave} RE, Wilson M, George KP, and Green DJ. Changes in vascular and cardiac function after prolonged strenuous exercise in humans. J Appl Physiol (1985) 105: 1562-1568, 2008.

24. de Jongh RT, Serne EH, RG IJ, de Vries G, and Stehouwer CD. Impaired microvascular function in obesity: implications for obesity-associated microangiopathy, hypertension, and insulin resistance. Circulation 109: 2529-2535, 2004. 
25. Gates PE, Boucher ML, Silver AE, Monahan KD, and Seals DR. Impaired flowmediated dilation with age is not explained by L-arginine bioavailability or endothelial asymmetric dimethylarginine protein expression. J Appl Physiol (1985) 102: 63-71, 2007.

26. Gill JM, Al-Mamari A, Ferrell WR, Cleland SJ, Packard CJ, Sattar N, Petrie JR, and Caslake MJ. Effects of prior moderate exercise on postprandial metabolism and vascular function in lean and centrally obese men. J Am Coll Cardiol 44: 2375-2382, 2004.

27. Gill JM, Al-Mamari A, Ferrell WR, Cleland SJ, Perry CG, Sattar N, Packard CJ, Caslake MJ, and Petrie JR. Effect of prior moderate exercise on postprandial metabolism in men with type 2 diabetes: heterogeneity of responses. Atherosclerosis 194: 134-143, 2007.

28. Green D. Point: Flow-mediated dilation does reflect nitric oxide-mediated endothelial function. J Appl Physiol 99: 1233-1234; discussion 1237-1238, 2005.

29. Green D, Cheetham C, Reed C, Dembo L, and O'Driscoll G. Assessment of brachial artery blood flow across the cardiac cycle: retrograde flows during cycle ergometry. J Appl Physiol (1985) 93: 361-368, 2002.

30. Harris RA, Padilla J, Hanlon KP, Rink LD, and Wallace JP. The flow-mediated dilation response to acute exercise in overweight active and inactive men. Obesity (Silver Spring) 16: 578$584,2008$.

31. Hashimoto M, Akishita M, Eto M, Ishikawa M, Kozaki K, Toba K, Sagara Y, Taketani Y, Orimo H, and Ouchi Y. Modulation of endothelium-dependent flow-mediated dilatation of the brachial artery by sex and menstrual cycle. Circulation 92: 3431-3435, 1995.

32. Hopkins ND, Stratton G, Tinken TM, McWhannell N, Ridgers ND, Graves LE, George K, Cable NT, and Green DJ. Relationships between measures of fitness, physical activity, body composition and vascular function in children. Atherosclerosis 204: 244-249, 2009.

33. Jaap AJ, Hammersley MS, Shore AC, and Tooke JE. Reduced microvascular hyperaemia in subjects at risk of developing type 2 (non-insulin-dependent) diabetes mellitus. Diabetologia 37: 214-216, 1994. 
34. Jenkins NT, Martin JS, Laughlin MH, and Padilla J. Exercise-induced Signals for

521 Vascular Endothelial Adaptations: Implications for Cardiovascular Disease. Current cardiovascular

522 risk reports 6: 331-346, 2012.

523 35. Johnson BD, Padilla J, and Wallace JP. The exercise dose affects oxidative stress and

524 brachial artery flow-mediated dilation in trained men. Eur J Appl Physiol 112: 33-42, 2012.

525 36. Khan F, Green FC, Forsyth JS, Greene SA, Morris AD, and Belch JJ. Impaired 526 microvascular function in normal children: effects of adiposity and poor glucose handling. $J$ Physiol $527 \quad 551: 705-711,2003$.

528 37. Kimm SY, Glynn NW, Obarzanek E, Kriska AM, Daniels SR, Barton BA, and Liu K. 529 Relation between the changes in physical activity and body-mass index during adolescence: a 530 multicentre longitudinal study. Lancet 366: 301-307, 2005.

531 38. Llewellyn TL, Chaffin ME, Berg KE, and Meendering JR. The relationship between 532 shear rate and flow-mediated dilation is altered by acute exercise. Acta Physiol (Oxf) 205: 394-402, 5332012.

534 39. Magkos F, Wright DC, Patterson BW, Mohammed BS, and Mittendorfer B. Lipid 535 metabolism response to a single, prolonged bout of endurance exercise in healthy young men. Am $J$ 536 Physiol Endocrinol Metab 290: E355-362, 2006.

537 40. Mahoney LT, Burns TL, Stanford W, Thompson BH, Witt JD, Rost CA, and Lauer 538 RM. Coronary risk factors measured in childhood and young adult life are associated with coronary 539 artery calcification in young adults: the Muscatine Study. J Am Coll Cardiol 27: 277-284, 1996.

540 41. Mancini GB, Yeoh E, Abbott D, and Chan S. Validation of an automated method for 541 assessing brachial artery endothelial dysfunction. Can J Cardiol 18: 259-262, 2002.

542 42. Mills A, Rosenberg M, Stratton G, Carter HH, Spence AL, Pugh CJ, Green DJ, and 543 Naylor LH. The effect of exergaming on vascular function in children. J Pediatr 163: 806-810, 5442013. 
43. Morris NM and Udry JR. Validation of a self-administered instrument to assess stage of

546

547

548

549

550

551

552

553

554

555

556

557

558

559

560

561

562

563

564

565

566

567

568

569

570

adolescent development. Journal of youth and adolescence 9: 271-280, 1980.

44. Motl RW, Dishman RK, Saunders R, Dowda M, Felton G, and Pate RR. Measuring enjoyment of physical activity in adolescent girls. Am J Prev Med 21: 110-117, 2001.

45. Mullen MJ, Kharbanda RK, Cross J, Donald AE, Taylor M, Vallance P, Deanfield JE, and MacAllister RJ. Heterogenous nature of flow-mediated dilatation in human conduit arteries in vivo: relevance to endothelial dysfunction in hypercholesterolemia. Circ Res 88: 145-151, 2001.

46. Padilla J, Harris RA, Fly AD, Rink LD, and Wallace JP. The effect of acute exercise on endothelial function following a high-fat meal. Eur J Appl Physiol 98: 256-262, 2006.

47. Padilla J, Simmons GH, Bender SB, Arce-Esquivel AA, Whyte JJ, and Laughlin MH. Vascular effects of exercise: endothelial adaptations beyond active muscle beds. Physiology (Bethesda) 26: 132-145, 2011.

48. Phillips LR, Parfitt G, and Rowlands AV. Calibration of the GENEA accelerometer for assessment of physical activity intensity in children. Journal of science and medicine in sport / Sports Medicine Australia 16: 124-128, 2013.

49. Ratel S, Lazaar N, Dore E, Baquet G, Williams CA, Berthoin S, Van Praagh E, Bedu M, and Duche P. High-intensity intermittent activities at school: controversies and facts. J Sports Med Phys Fitness 44: 272-280, 2004.

50. Riddoch CJ, Mattocks C, Deere K, Saunders J, Kirkby J, Tilling K, Leary SD, Blair SN, and Ness AR. Objective measurement of levels and patterns of physical activity. Arch Dis Child 92: 963-969, 2007.

51. Roche DM, Rowland TW, Garrard M, Marwood S, and Unnithan VB. Skin microvascular reactivity in trained adolescents. Eur J Appl Physiol 108: 1201-1208, 2010.

52. Rognmo O, Bjornstad TH, Kahrs C, Tjonna AE, Bye A, Haram PM, Stolen T, Slordahl SA, and Wisloff U. Endothelial function in highly endurance-trained men: effects of acute exercise. J Strength Cond Res 22: 535-542, 2008. 
571 53. Sedgwick MJ, Morris JG, Nevill ME, and Barrett LA. Effect of repeated sprints on 572 postprandial endothelial function and triacylglycerol concentrations in adolescent boys. J Sports Sci $5731-11,2014$.

574 54. Sedgwick MJ, Morris JG, Nevill ME, Tolfrey K, Nevill A, and Barrett LA. Effect of 575 exercise on postprandial endothelial function in adolescent boys. Br J Nutr 1-9, 2012.

576 55. Seip RL, and Semenkovich CF. Skeletal muscle lipoprotein lipase: molecular regulation 577 and physiological effects in relation to exercise. Exerc Sport Sci Rev 26: 191-218, 1998.

578 56. Serne EH, Gans RO, ter Maaten JC, Tangelder GJ, Donker AJ, and Stehouwer CD. 579 Impaired skin capillary recruitment in essential hypertension is caused by both functional and 580 structural capillary rarefaction. Hypertension 38: 238-242, 2001.

581 57. Slaughter MH, Lohman TG, Boileau RA, Horswill CA, Stillman RJ, Van Loan MD, 582 and Bemben DA. Skinfold equations for estimation of body fatness in children and youth. Hum 583 Biol 60: 709-723, 1988.

584 58. Stary HC. Evolution and progression of atherosclerotic lesions in coronary arteries of 585 children and young adults. Arteriosclerosis 9: I19-32, 1989.

586 59. Suwaidi JA, Hamasaki S, Higano ST, Nishimura RA, Holmes DR, Jr., and Lerman A. 587 Long-term follow-up of patients with mild coronary artery disease and endothelial dysfunction. 588 Circulation 101: 948-954, 2000.

589 60. Thackray AE, Barrett LA, and Tolfrey K. Acute High-Intensity Interval Running 590 Reduces Postprandial Lipemia in Boys. Med Sci Sports Exerc 45: 1277-1284, 2013.

591 61. Thijssen DH, Black MA, Pyke KE, Padilla J, Atkinson G, Harris RA, Parker B, 592 Widlansky ME, Tschakovsky ME, and Green DJ. Assessment of flow-mediated dilation in 593 humans: a methodological and physiological guideline. Am J Physiol Heart Circ Physiol 300: H2$59412,2011$.

595 62. Thijssen DH, Bullens LM, van Bemmel MM, Dawson EA, Hopkins N, Tinken TM, 596 Black MA, Hopman MT, Cable NT, and Green DJ. Does arterial shear explain the magnitude of 
flow-mediated dilation?: a comparison between young and older humans. Am J Physiol Heart Circ Physiol 296: H57-64, 2009.

63. Thijssen DH, Dawson EA, Black MA, Hopman MT, Cable NT, and Green DJ. Brachial artery blood flow responses to different modalities of lower limb exercise. Med Sci Sports Exerc 41: 1072-1079, 2009.

64. Tinken TM, Thijssen DH, Hopkins N, Black MA, Dawson EA, Minson CT, Newcomer SC, Laughlin MH, Cable NT, and Green DJ. Impact of shear rate modulation on vascular function in humans. Hypertension 54: 278-285, 2009.

65. Tinken TM, Thijssen DH, Hopkins N, Dawson EA, Cable NT, and Green DJ. Shear stress mediates endothelial adaptations to exercise training in humans. Hypertension 55: 312-318, 2010.

66. Tolfrey K, Bentley C, Goad M, Varley J, Willis S, and Barrett L. Effect of energy expenditure on postprandial triacylglycerol in adolescent boys. Eur J Appl Physiol 112: 23-31, 2012.

67. Tolfrey K, Doggett A, Boyd C, Pinner S, Sharples A, and Barrett L. Postprandial triacylglycerol in adolescent boys: a case for moderate exercise. Med Sci Sports Exerc 40: 10491056, 2008.

68. Tolfrey K, Engstrom A, Murphy C, Thackray A, Weaver R, and Barrett LA. Exercise energy expenditure and postprandial lipemia in girls. Med Sci Sports Exerc 46: 239-246, 2014.

69. Trost SG, Pate RR, Sallis JF, Freedson PS, Taylor WC, Dowda M, and Sirard J. Age and gender differences in objectively measured physical activity in youth. Med Sci Sports Exerc 34: 350-355, 2002.

70. Tyldum GA, Schjerve IE, Tjonna AE, Kirkeby-Garstad I, Stolen TO, Richardson RS, and Wisloff U. Endothelial dysfunction induced by post-prandial lipemia: complete protection afforded by high-intensity aerobic interval exercise. J Am Coll Cardiol 53: 200-206, 2009. 
622 71. Vogel RA, Corretti MC, and Plotnick GD. Effect of a single high-fat meal on endothelial 623 function in healthy subjects. Am J Cardiol 79: 350-354, 1997.

624 72. Wallace JP, Johnson B, Padilla J, and Mather K. Postprandial lipaemia, oxidative stress 625 and endothelial function: a review. International journal of clinical practice 64: 389-403, 2010.

626 73. Yelling M, Lamb K, and Swaine I. Validity of a Pictorial Perceived Exertion Scale for 627 Effort Estimation and Effort Production During Stepping Exercise in Adolescent Children. 628 European Physical Education Review 8: 157-175, 2002.

629 74. Zhang JQ, Thomas TR, and Ball SD. Effect of exercise timing on postprandial lipemia 630 and HDL cholesterol subfractions. J Appl Physiol 85: 1516-1522, 1998.

631

632

633

634

635

636

637

638

639

640

641

642 
644

645

646

Table 1 Participant characteristics.

\begin{tabular}{lcccc}
\hline & Boys $(n=10)$ & Girls $(n=10)$ & $P$ value & $E S$ \\
\hline Age $(\mathrm{y})$ & $14.8 \pm 0.2$ & $14.1 \pm 0.9$ & 0.06 & 1.07 \\
Body mass $(\mathrm{kg})$ & $61.1 \pm 11.9$ & $54.5 \pm 9.3$ & 0.19 & 0.62 \\
Stature $(\mathrm{m})$ & $1.69 \pm 0.07$ & $1.61 \pm 0.09$ & 0.04 & 0.99 \\
Body fat $(\%)$ & $10 \pm 4$ & $20 \pm 4$ & $<0.001$ & 2.50 \\
$\dot{V} \mathrm{O}_{2 \max }\left(\mathrm{L} \cdot \mathrm{min}^{-1}\right)$ & $2.76 \pm 0.54$ & $2.03 \pm 0.27$ & 0.001 & 1.71 \\
$\dot{V} \mathrm{O}_{2 \max }\left(\mathrm{mL}^{-1} \min ^{-1} \cdot \mathrm{kg}^{-1}\right)$ & $45.5 \pm 6.4$ & $37.8 \pm 4.5$ & 0.01 & 1.39 \\
$\mathrm{GET}\left(\mathrm{L} \cdot \mathrm{min}^{-1}\right)$ & $1.40 \pm 0.25$ & $1.09 \pm 0.20$ & 0.001 & 1.37 \\
$\mathrm{GET}\left(\% \dot{\mathrm{V}} \mathrm{O}_{2 \max }\right)$ & $51 \pm 6$ & $54 \pm 7$ & 0.39 & 0.46
\end{tabular}

647

648

649

650

651

652

653

654

655

656

657

658

659

660

661

662

663

664

665

666

667

668

669

$\dot{V} \mathrm{O}_{2}$, oxygen uptake; GET, gas exchange threshold; $E S=$ effect size. Data presented as mean $\pm \mathrm{SD}$. 
670 Table 2: Accelerometer and food diary data during the 48 hours preceding each trial

\begin{tabular}{|c|c|c|c|c|c|c|}
\hline & $\mathrm{CON}$ & MIE & HIIE & $\begin{array}{c}\text { MIE vs. CON } \\
95 \% \mathrm{CI}\end{array}$ & $\begin{array}{c}\text { HIIE vs. CON } \\
95 \% \mathrm{CI}\end{array}$ & $\begin{array}{c}\text { HIIE vs. MIE } \\
95 \% \mathrm{CI}\end{array}$ \\
\hline Moderate-vigorous activity (min day $^{-1}$ ) & $75 \pm 30$ & $73 \pm 36$ & $75 \pm 27$ & -35 to 19 & -37 to 23 & -18 to 24 \\
\hline Total energy intake $\left(\mathrm{kcal} \mathrm{day}^{-1}\right)$ & $1862 \pm 427$ & $1980 \pm 388$ & $2027 \pm 551$ & -122 to 245 & -134 to 455 & -171 to 369 \\
\hline Energy from carbohydrates (\%) & $46 \pm 5$ & $47 \pm 5$ & $45 \pm 5$ & -1 to 5 & -3 to 3 & -5 to 2 \\
\hline Energy from fat $(\%)$ & $37 \pm 6$ & $36 \pm 4$ & $37 \pm 6$ & -5 to 2 & -5 to 2 & -4 to 4 \\
\hline Energy from protein $(\%)$ & $17 \pm 4$ & $17 \pm 3$ & $18 \pm 3$ & -4 to 2 & -1 to 3 & 0 to 4 \\
\hline
\end{tabular}

CON, control trial; MIE, moderate-intensity exercise trial; HIIE, high-intensity interval exercise trial $95 \% \mathrm{CI}=95 \%$ confidence limits for the true difference

Data have been pooled as ANOVA analysis revealed no main effect for sex

676

677

678

679

680

681

682

683

684

685 
Table 3 Physiological and perceptual responses to exercise conditions.

\begin{tabular}{|c|c|c|c|c|}
\hline & MIE & HIIE & $P$ value & ES \\
\hline \multicolumn{5}{|l|}{ Boys } \\
\hline Mean HR (b·min $\left.{ }^{-1}\right)$ & $117 \pm 7$ & $144 \pm 4$ & $<0.001$ & 4.74 \\
\hline Mean $\operatorname{HR}\left(\% \mathrm{HR}_{\max }\right)$ & $63 \pm 4$ & $77 \pm 3$ & $<0.001$ & 3.96 \\
\hline Mean $\dot{V} \mathrm{O}_{2}\left(\mathrm{~L} \cdot \mathrm{min}^{-1}\right)$ & $1.25 \pm 0.19$ & $1.59 \pm 0.25$ & $<0.001$ & 1.53 \\
\hline Mean $\dot{V} \mathrm{O}_{2}\left(\% \dot{V} \mathrm{O}_{2 \max }\right)$ & $46 \pm 7$ & $58 \pm 4$ & $<0.001$ & 2.10 \\
\hline RER & $0.89 \pm 0.04$ & $1.05 \pm 0.04$ & $<0.001$ & 4.00 \\
\hline RPE & $4 \pm 1$ & $8 \pm 1$ & $<0.001$ & 4.00 \\
\hline PACES & $53 \pm 15$ & $64 \pm 7$ & 0.08 & 0.94 \\
\hline Work performed (kJ) & $136 \pm 24$ & $136 \pm 24$ & - & - \\
\hline Energy Expenditure $(\mathrm{kJ})$ & $635 \pm 100$ & - & - & - \\
\hline \multicolumn{5}{|l|}{ Girls } \\
\hline Mean HR $\left(b \cdot \min ^{-1}\right)$ & $144 \pm 13$ & $158 \pm 12$ & 0.01 & 1.12 \\
\hline Mean HR $\left(\% \mathrm{HR}_{\max }\right)$ & $74 \pm 6$ & $81 \pm 5$ & 0.01 & 1.27 \\
\hline Mean $\dot{V} \mathrm{O}_{2}\left(\mathrm{~L} \cdot \mathrm{min}^{-1}\right)$ & $1.10 \pm 0.09$ & $1.26 \pm 0.11$ & $<0.001$ & 1.59 \\
\hline Mean $\dot{V} \mathrm{O}_{2}\left(\% \dot{V} \mathrm{O}_{2 \max }\right)$ & $55 \pm 4$ & $62 \pm 5$ & $<0.001$ & 1.55 \\
\hline RER & $0.89 \pm 0.05$ & $1.04 \pm 0.02$ & $<0.001$ & 3.94 \\
\hline RPE & $5 \pm 2$ & $7 \pm 1$ & 0.01 & 1.26 \\
\hline PACES & $54 \pm 10$ & $59 \pm 7$ & 0.17 & 0.58 \\
\hline Work performed (kJ) & $109 \pm 11$ & $109 \pm 11$ & - & - \\
\hline Energy Expenditure $(\mathrm{kJ})$ & $700 \pm 82$ & - & - & - \\
\hline
\end{tabular}

688

689

690

691

692

693

694

695

696

697

698

699

700

701

702

703

704

705

706

707

708

$\mathrm{HR}$, heart rate; $\dot{V} \mathrm{O}_{2}$, oxygen uptake; MIE, moderate-intensity exercise trial; HIIE, high-intensity exercise trial; $E S=$ effect size. Data presented as mean \pm SD. $n=10$ for boys and girls apart from mean HR where $n=8$ due to loss of telemetric data. 


\section{FIGURES}

710

711

712

713

714

715

716

717

718

719

720

721

722

723

724

725

726

727

728

729

730

731

732

733

Figure 3. Mean changes in flow mediated dilation (A), area under the curve until peak dilation for shear rate (B), baseline arterial diameter (C) and peak microvascular perfusion (D), for the control $(\mathrm{O})$, moderate- $(\boldsymbol{\Delta})$ and high-( $\mathbf{\square})$ intensity exercise conditions. Error bars represent the standard deviation. The high fat meal is represented by the black rectangle. Statistical significance between conditions at the same timepoint are described as follows: ${ }^{*}$ HIIE vs CON; ${ }^{*}$ HIIE vs MIE; ${ }^{\dagger}$ MIE vs CON. Within-condition significant difference from baseline: ${ }^{\S} \mathrm{HIIE} ;{ }^{\star} \mathrm{CON}$. Refer to text for specific $P$ values.

734

735 

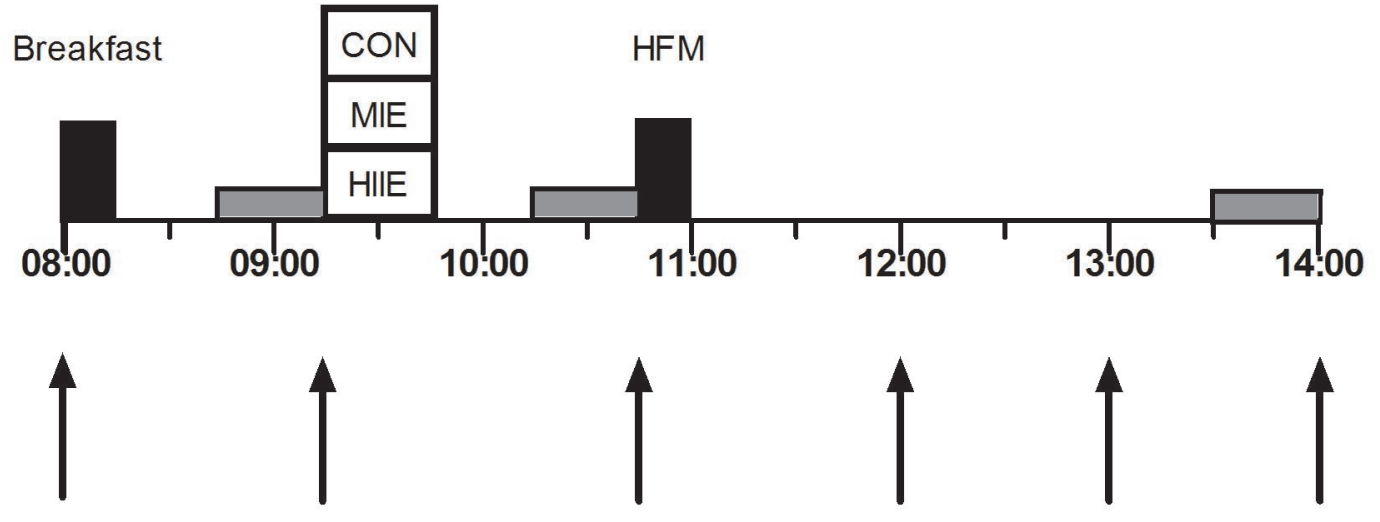
A

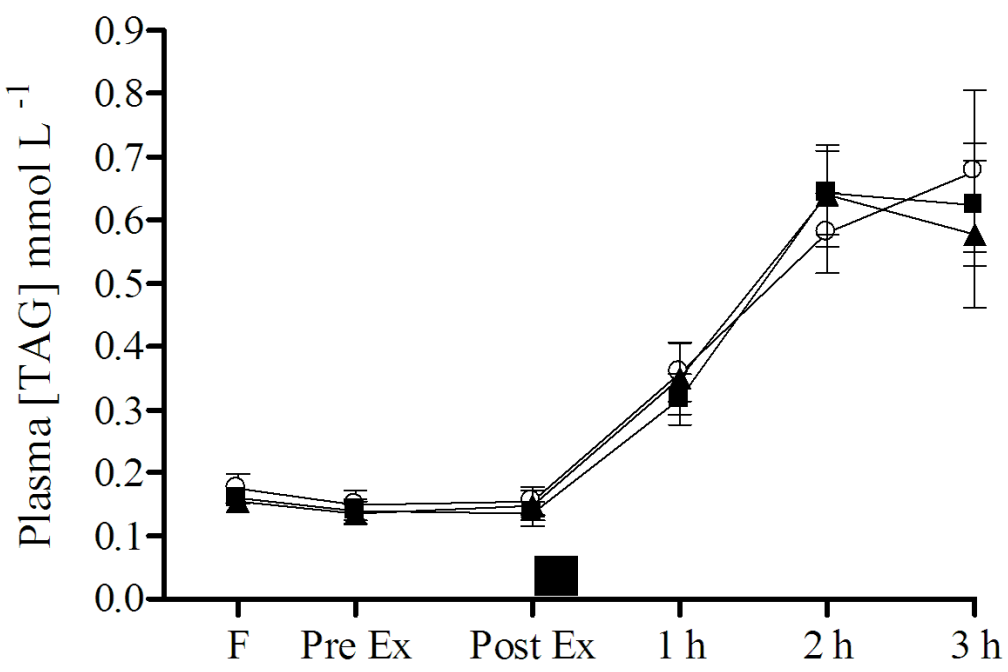

B

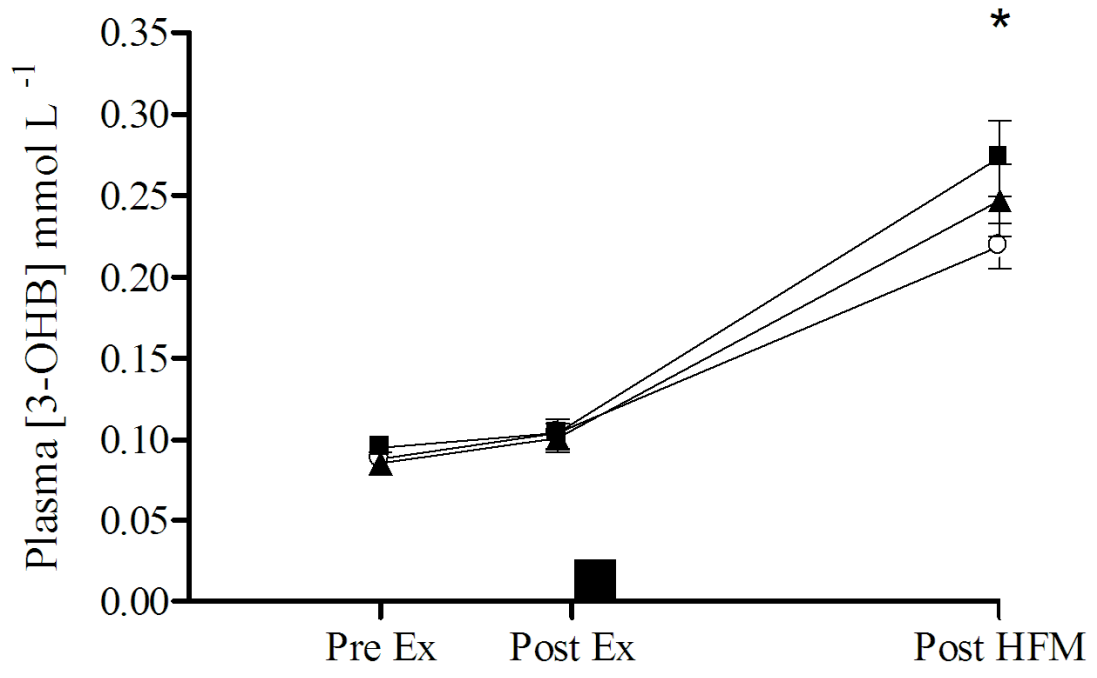

C

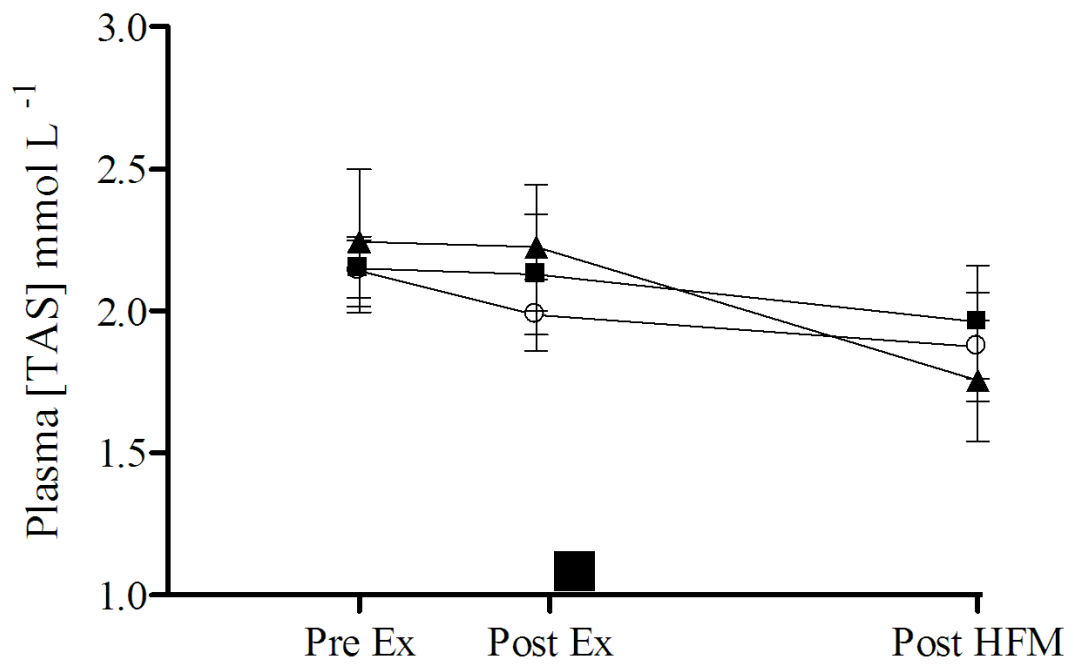



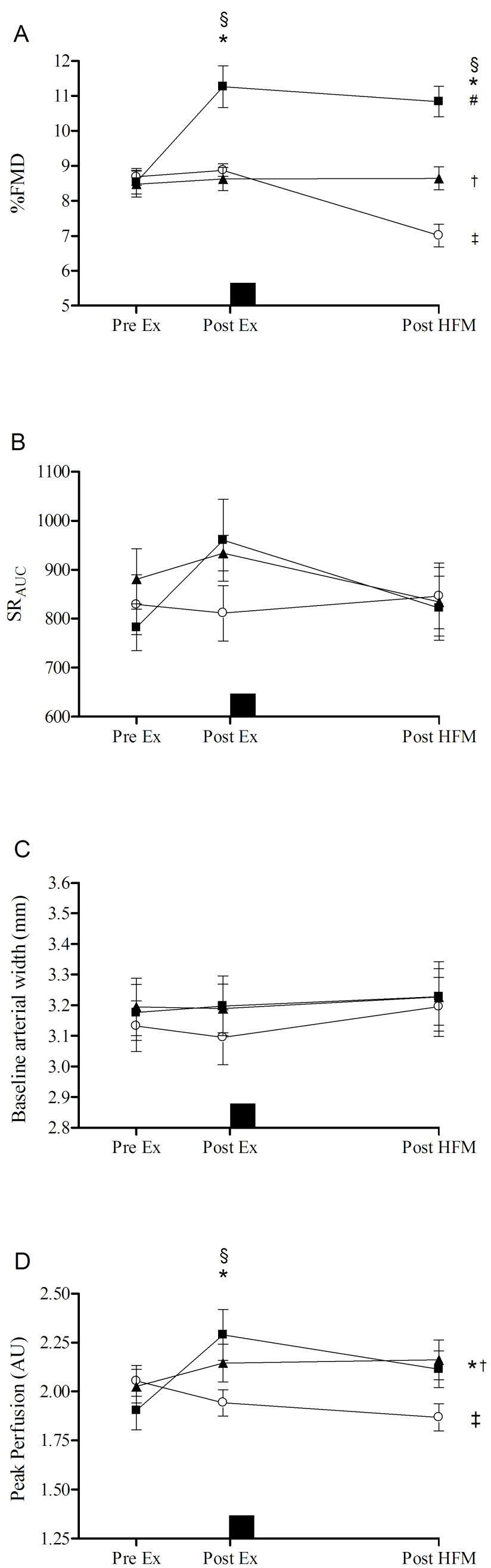